\title{
Correction to: Combined whole cell wall analysis and streamlined in silico carbohydrate-active enzyme discovery to improve biocatalytic conversion of agricultural crop residues
}

Jeffrey P. Tingley ${ }^{1,2}$, Kristin E. Low ${ }^{1}$, Xiaohui Xing ${ }^{1}$ and D. Wade Abbott ${ }^{1,2^{*}}$

\section{Correction to: Biotechnol Biofuels (2021) 14:16} https://doi.org/10.1186/s13068-020-01869-8

Following publication of the original article [1], the authors noticed an error in the figures. It was noticed that due to typesetter error and file conversion, incorrect versions of the figures were published. The corrected Figs. 1, 2, 3, 4, 5 are given below.

The original article [1] has been updated. is not included in the article's Creative Commons licence and your intended use is not permitted by statutory regulation or exceeds the permitted use, you will need to obtain permission directly from the copyright holder. To view a copy of this licence, visit http://creativeco mmons.org/licenses/by/4.0/. The Creative Commons Public Domain Dedication waiver (http://creativecommons.org/publicdomain/ zero/1.0/) applies to the data made available in this article, unless otherwise stated in a credit line to the data. 


\section{Hemicelluloses}
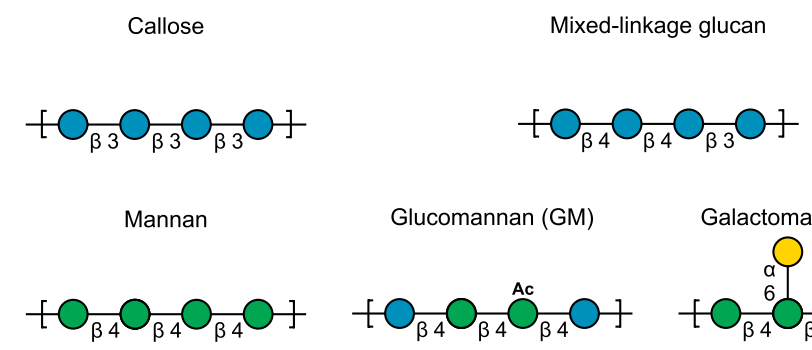

Glucomannan (GM)

Galactomannan (GaM)

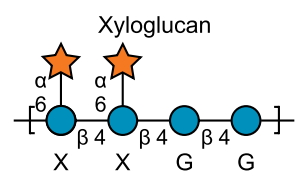

$$
\text { Xylan }
$$
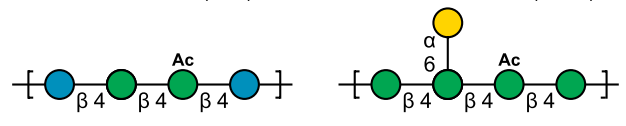

Galactoglucomannan (GGM)

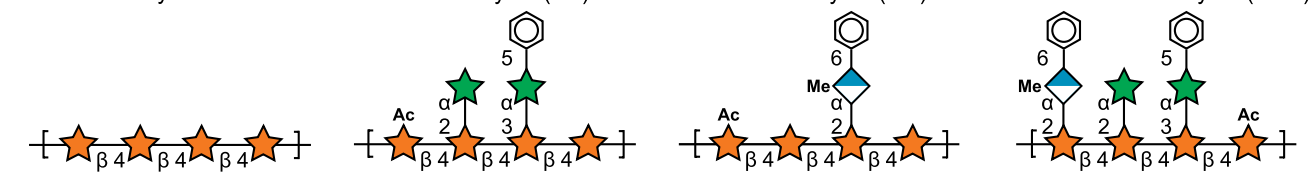

\section{Pectins}

Homogalacturonan (HG)
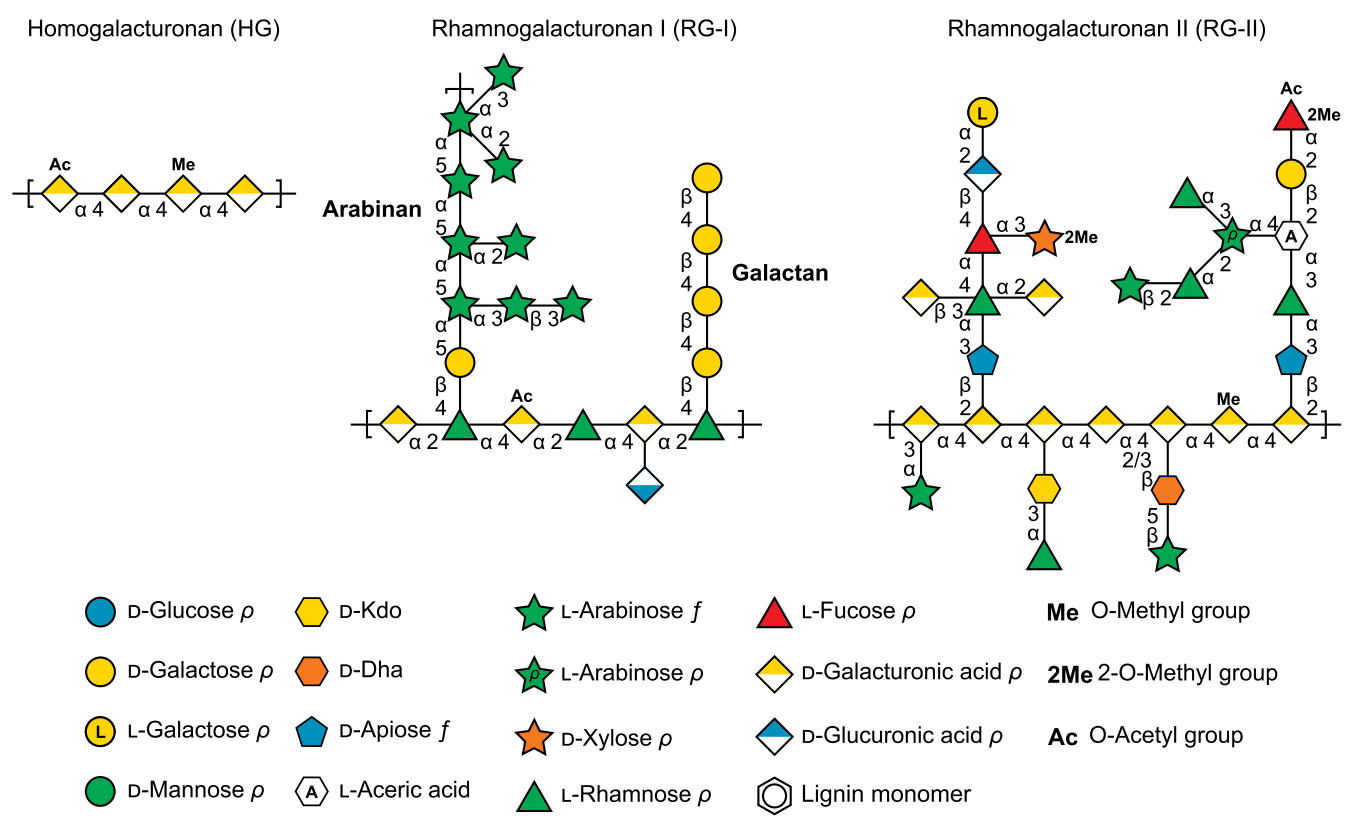

$\sim$ L-Arabinose $f$

$\triangle$ L-Fucose $\rho$

Me O-Methyl group

饮 L-Arabinose $\rho$

$\diamond$ D-Galacturonic acid $\rho$

2Me 2-O-Methyl group

$\hat{W}$ D-Xylose $\rho$

$\Leftrightarrow$ D-Glucuronic acid $\rho$

Ac O-Acetyl group

Fig. 1 Cartoon schematic of non-cellulosic plant cell wall polysaccharides. Representative schematics chosen for xyloglucan [225], mannans and xylans [226], and pectins [24, 114]. Monosaccharide symbols follow the Symbol Nomenclature for Glycans [227] 


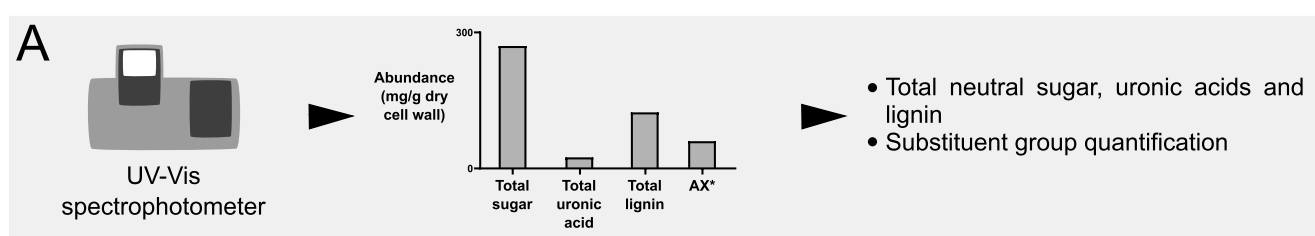

B

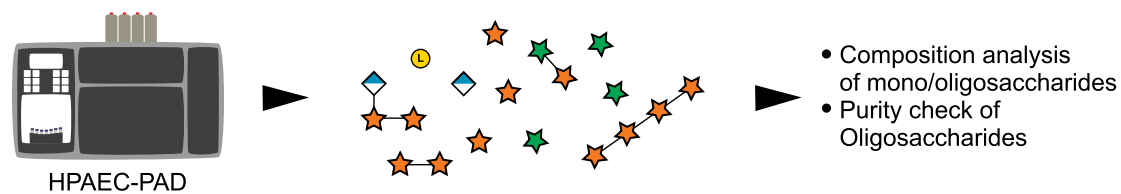

C

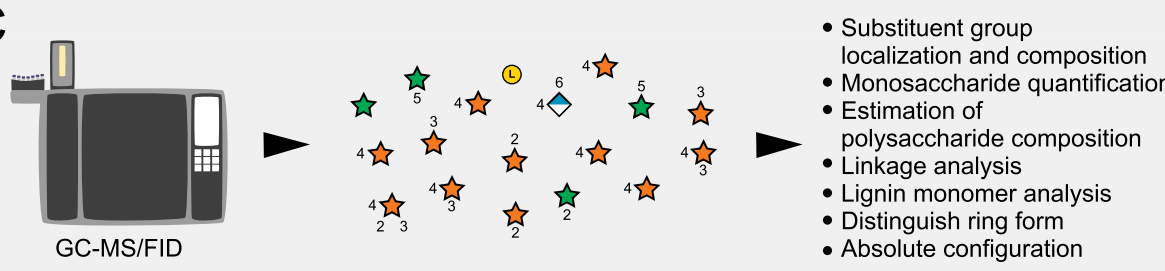

D

(

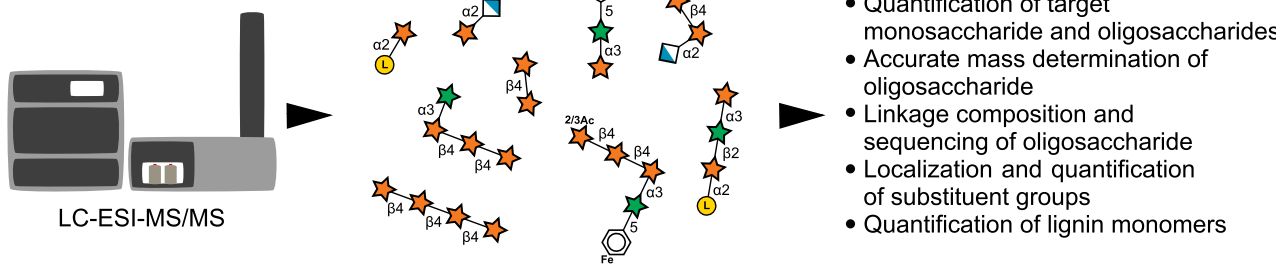

$E$

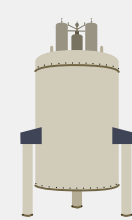

NMR

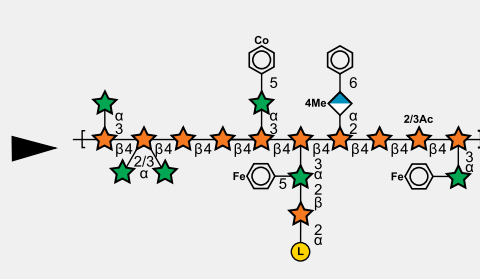

(0) 2 ? $^{3}$

- Anomeric configuration

- Absolute configuration

- Distinguish ring form

- Location and degree of substitution

- Monosaccharide linkage sequencing

of complex carbohydrates

- Monosaccharide, oligosaccharide,

and polysaccharide profiling

- Lignin monomer composition

- Carbohydrate-lignin interaction

F

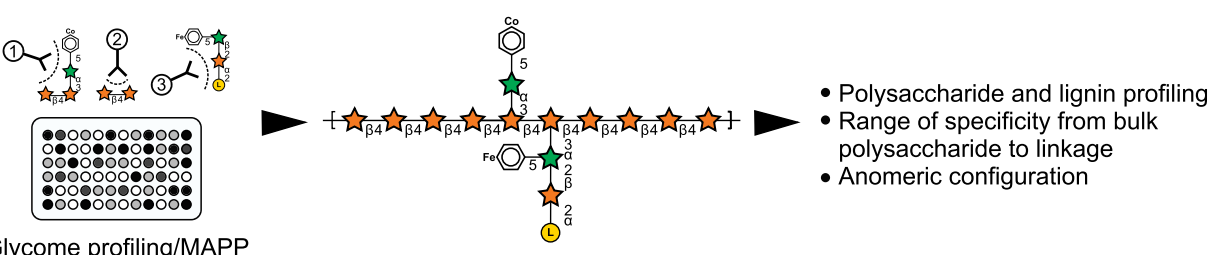

Glycome profiling/MAPP

\section{$\triangle$ D-Glucuronic acid $\rho$ \\ (L) L-Galactose $\rho$ \\ (O) Lignin monomer}

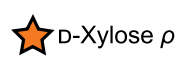

Ac O-Acetyl group

Fe Feruloyl residue


Me O-Methyl group

Co $\rho$-Coumaroyl residue

Fig. 2 Analytical methods for total cell wall analysis. a UV/Vis spectrophotometer colorimetric assays. AX*: total arabinoxylan can be determined through commercially available kit; b HPAEC-PAD; c GC-MS/FID; d LC-ESI-MS/MS; e NMR; and f Immunological methods, such as Glycome profiling and MAPP. Corn GAX was used as a model polysaccharide to demonstrate representative structural information that could be inferred by each method [28] 
a<smiles>[R]O[CH+][C@H]1CCC[C@H](O[C@@H]2CCCO2)OC1</smiles>

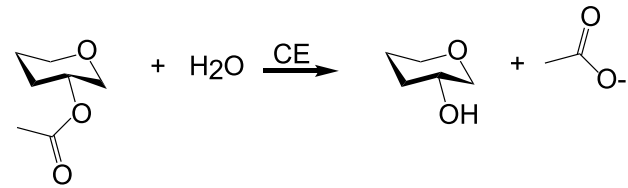

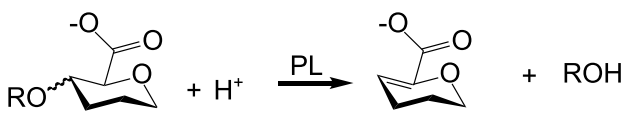

$3 \mathrm{HC}-\mathrm{O}$
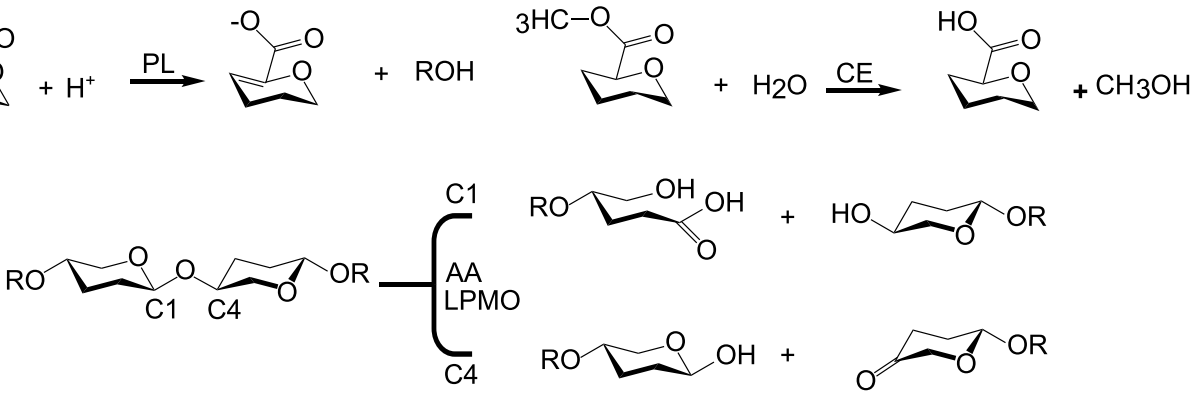

b

Homogalacturonan (HG)

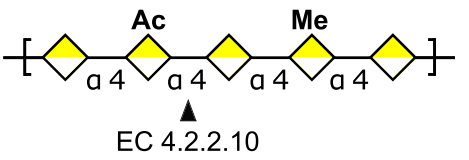

Cellulose

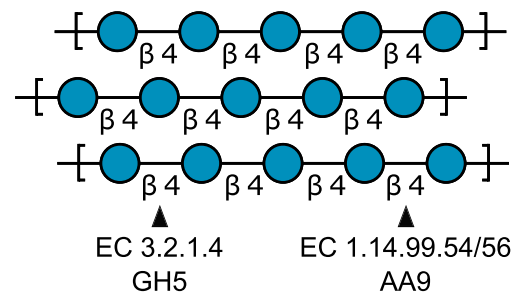

Corn Glucuronoarabinoxylan (GAX)

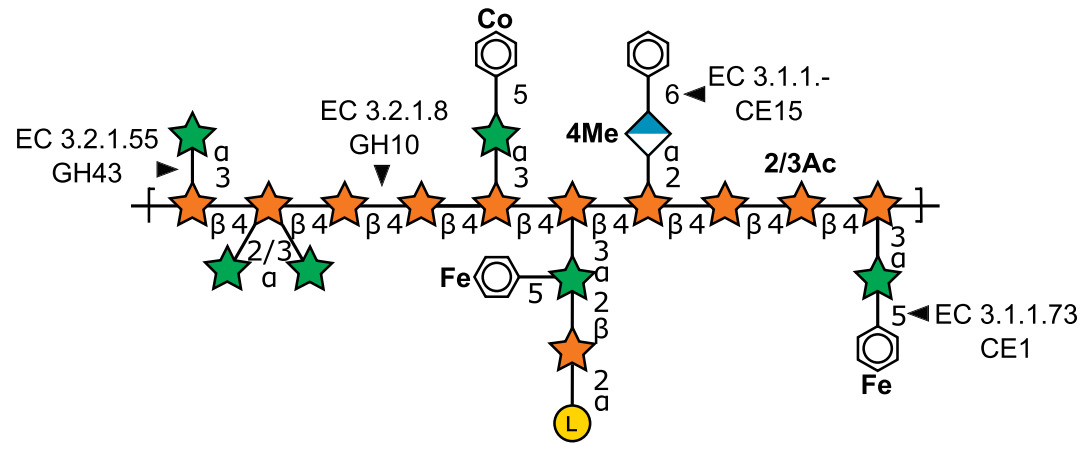

\section{$\diamond$ D-Galacturonicacid $\rho \bigcirc$ D-Glucose $\rho \quad \widehat{D}$-Xylose $\rho \quad$ Ac O-Acetyl group $\diamond$ D-Glucuronicacid $\rho$ (L) L-Galactose $\rho$ L-Arabinose $f \quad$ Me O-Methyl group \\ (0) Lignin monomer Fe Feruloyl residue Co $\rho$-Coumaroyl residue}

Fig. 3 CAZyme depolymerization mechanisms and specificities. a Simplified reaction schematics are shown of a glycoside hydrolase (GH), polysaccharide lyase (PL), carbohydrate esterases (CEs) acetyl (top) and methyl (bottom), and the auxiliary activities (AA) of LPMOs active on C1 and C4. b CAZyme-targeted bonds of plant cell wall polysaccharides homogalacturonan (HG), cellulose, and corn GAX [28]) are shown, with example CAZy family and enzyme class (EC) numbers as indicated 
a

$\square$ Subfamily $2 \square$ Subfamily $9 \square$ Subfamily 19
$\square$ Subfamily $7 \square$ Subfamily $15 \square$ Subfamily 21

1

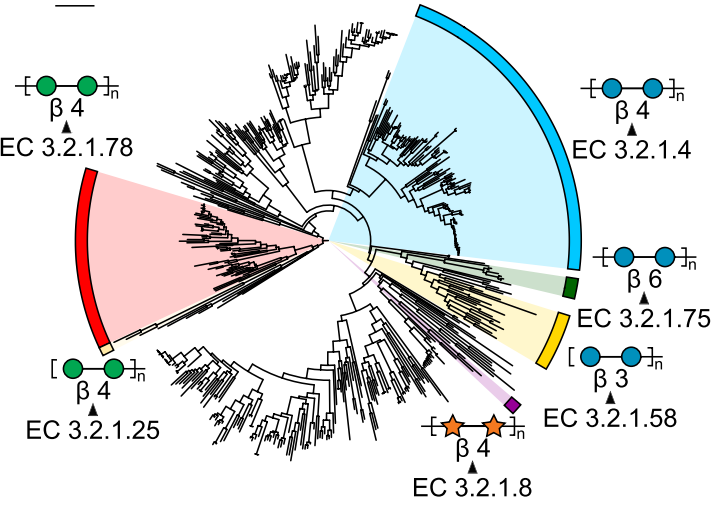

b

$\square$ Subfamily $4 \quad \square$ Subfamily $24 \square$ Subfamily 30 $\square$ Subfamily $11 \square$ Subfamily 16

1

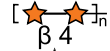
EC 3.2 .37

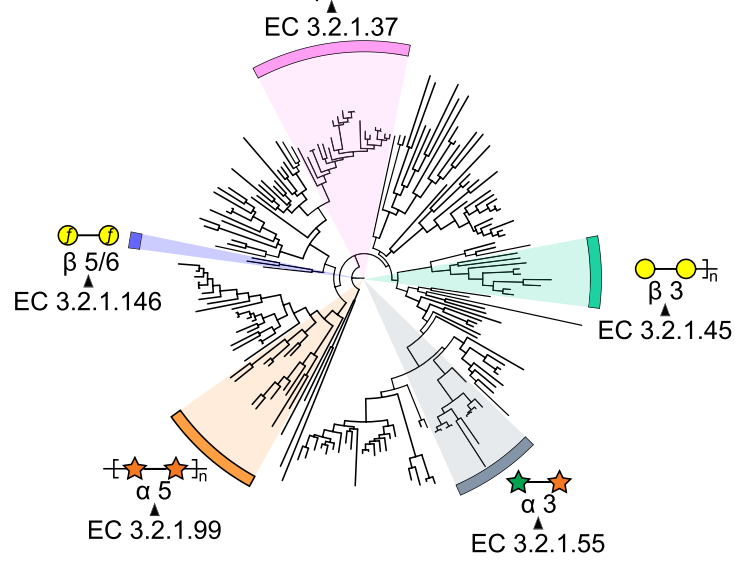

OD-Glucose $\rho$ OD-Mannose $\rho$ DD-Xylose $\rho$

OD-Galactose $\rho \oplus \mathrm{D}$-Galactose $f$ L-Arabinose $f$

Fig. 4 Polyspecific CAZy families GH5 and GH43. Phylogenetic trees were built using SACCHARIS [195] with characterized sequences for a GH5 and b GH43 CAZy families. Annotations were generated using ITOL [228]. Enzyme activities, for example, subfamilies, are labeled with the corresponding EC numbers, and targeted substrates are illustrated by cartoons following the Symbol Nomenclature for Glycans [227] 


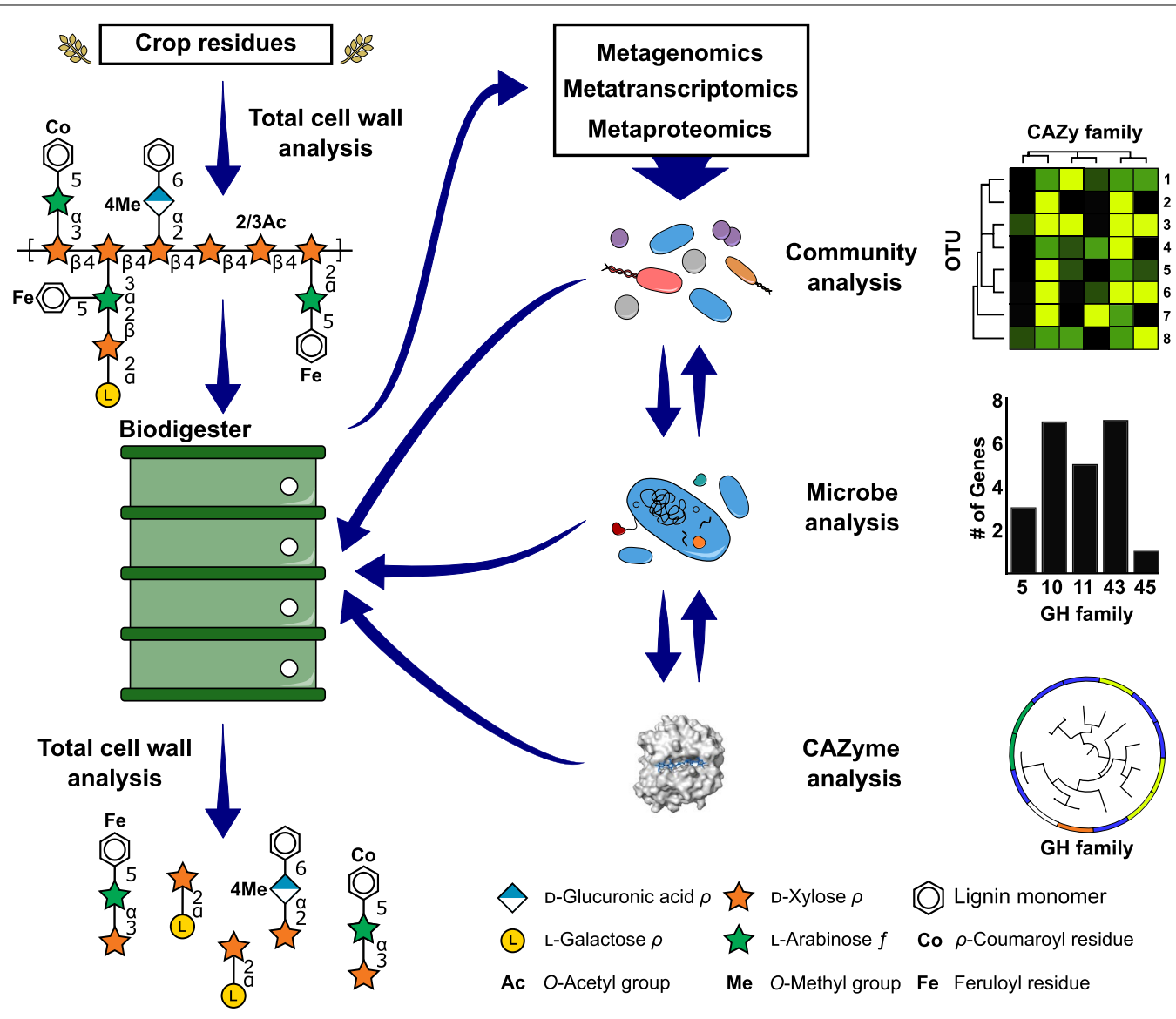

Fig. 5 Combinatorial assessment of cell wall structure and investigation of microbial CAZyme function. The integration of analytical methods can be implemented to provide a comprehensive experimental workflow to improve bioconversion of agriculture residues. Crop residues can be studied prior to or after processing using total cell wall analysis. Information on the structure of waste residues can be compared to starting material to determine recalcitrant structures that are limiting the efficiency of bioconversion. The microbial ecosystem of biodigesters can be studied using -omics techniques, such as metagenomics, metatranscriptomics, and metaproteomics, to define the structure and function at the community, microbe, and CAZyme levels. Information gathered using these techniques can inform optimized conditions or identify lacking catalytic functions in the reaction cascade. Microbial communities, microorganisms, and CAZymes can be deployed back into production processes to augment inefficent or absent catalytic reactions and improve biofuel production. Surface representation of enzyme structure (white) was generated using PyMOL [229] (PDB ID: 2CKR), with cellotetrose ligand illustrated in sticks (blue)

\section{Author details}

${ }^{1}$ Lethbridge Research and Development Centre, Agriculture and Agri-Food Canada, 5403-1st Avenue South, Lethbridge, AB T1J 4B1, Canada. ${ }^{2}$ Department of Biochemistry, University of Lethbridge, Lethbridge, AB T1K 6T5, Canada.

Received: 20 January 2021 Accepted: 20 January 2021

Published online: 08 February 2021

\section{Reference}

1. Tingley JP, Low KE, Xing X, Abbott DW. Combined whole cell wall analysis and streamlined in silico carbohydrate-active enzyme discovery to improve biocatalytic conversion of agricultural crop residues. Biotechnol Biofuels. 2021;14:16. https://doi.org/10.1186/s13068-020-01869-8.

\section{Publisher's Note}

Springer Nature remains neutral with regard to jurisdictional claims in published maps and institutional affiliations. 